\title{
FARMÁCIA
}

\section{O USO INDISCRIMINADO DOS ANTI-INFLAMATÓRIOS NÃO ESTEROIDAIS (AINES)}

\author{
DOI: http://dx.doi.org/0.31072/rcf.v8i2.589 \\ THE INDISCRIMINATED USE OF NON-STEROID ANTI-INFLAMMATORY (NSAID)
}

Alline Correia Sandoval ${ }^{1}$; Dione Rodrigues Fernandes ${ }^{2}$; Ederson Aparecido da Silva ${ }^{3}$; André

Tomaz Terra Júnior ${ }^{4}$.

RESUMO: Os anti-inflamatórios não esteroides (AINEs) constituem uma das classes de fármacos mais prescritas em todo mundo. Atualmente no mercado farmacêutico existem mais de 50 diferentes tipos de AINEs, são utilizados no tratamento da dor aguda, moderada e crônica decorrente de processo inflamatório. Os AINEs possuem três tipos de ações diferentes: Anti-inflamatória, Analgésica e Antipirética. Esse processo ocorre devido a inibição da enzima ciclooxigenase (COX) que é responsável pela hidrolise do Ácido Araquidônico (AA) tornando-o em diversos mediadores lipídicos denominados de prostaglandinas (PGs) e Tromboxanos (TX2), essas substâncias tem importante função no equilíbrio da proteção da mucosa gástrica (citoproteção gástrica), fisiologia renal, gestação e agregação plaquetária. Com a inibição das isoformas foram criados dois tipos de subgrupos de AINEs os seletivos e os não seletivos, sendo essa classificação de acordo com a sua seletividade. Os AINEs seletivos são mais antigos e designados como tradicionais ou convencionais. Os AINEs não seletivos diminuem a produção de todas as PGs constitutivas ou não. Já os seletivos da COX2 atuam inibindo a apenas PGs deletérias, que fazem parte do processo inflamatório e, preservando as $P G$ que fazem proteção da mucosa do TGI e da perfusão renal. Objetivo desse estudo é verificar as características dos anti-inflamatórios não esteroides, como agentes terapêuticos no processo inflamatório e as principais consequências do uso indiscriminado. Foram realizadas buscas em artigos científicos no período do ano 2000 - 2017 para a realização deste trabalho.

Descritores (DeCS)5: Anti-inflamatórios não-esteroides. Processo inflamatório. COX-1. COX2.

ABSTRACT: Non-steroidal anti-inflammatory drugs (NSAIDs) are one of the most prescribed classes of drugs in the world. Currently in the pharmaceutical market there are more than 50

\footnotetext{
1 Discente do curso de Farmácia da Faculdade de Educação e Meio Ambiente - FAEMA. E-mail: bamc fest@hotmail.com. ORCID: https://orcid.org/0000-0002-4102-9593;

2 Bacharel em Farmácia pela Faculdade de Educação e Meio Ambiente - FAEMA. E-mail: dionefernandes.claro@gmail.com. ORCID: https://orcid.org/0000-0001-7349-3246;

3 Discente do curso de Farmácia da faculdade de Educação e Meio Ambiente - FAEMA. E-mail: eder_mattedi@hotmail.com. ORCID: https://orcid.org/0000-0001-8019-7357;

${ }^{4}$ Mestre em Oncologia Clínica, Terapia Celular e Células troncos pela Faculdade de Medicina de Ribeirão Preto -FMRP/USP. Docente do curso de graduação em Farmácia da Faculdade de Educação e Meio Ambiente FAEMA. E-mail: andretomazfaema@gmail.com. ORCID: https://orcid.org/0000-0001-7365-5284.

${ }^{5}$ Vide http://decs.bvs.br.
} 
different types of NSAIDs, they are used in the treatment of acute, moderate and chronic pain due to inflammatory process. NSAIDs have three different types of actions: Anti-inflammatory, Analgesic and Antipyretic. This process occurs due to the inhibition of the cyclooxygenase (COX) enzyme that is responsible for the hydrolysis of Arachidonic Acid (AA), making it into several lipid mediators called prostaglandins (PGs) and Thromboxanes (TX2). protection of the gastric mucosa (gastric cytoprotection), renal physiology, gestation and platelet aggregation. With the inhibition of the isoforms, two types of NSAID subgroups were created: the selective and the non-selective NSAIDs, being this classification according to their selectivity. Selective NSAIDs are older and designated as traditional or conventional. Nonselective NSAIDs decrease the production of all constitutive PGs or not. On the other hand, the selective COX2 acts to inhibit only deleterious PGs, which are part of the inflammatory process and, preserving the PGs that protect the mucosa of the GIT and renal perfusion. Aim of this study is to verify the characteristics of non-steroidal anti-inflammatory drugs as therapeutic agents in the inflammatory process and the main consequences of indiscriminate use. Researches were carried out in scientific articles from 2000 to 2017 to carry out this work.

Descriptors: Anti-inflammatory nonsteroidal. Inflammation. COX-1. COX-2.

\section{INTRODUÇÃO}

Os anti-inflamatórios não esteroides AINEs constituem uma das classes de fármacos mais prescritas em todo mundo (1). Atualmente no mercado farmacêutico existem mais de 50 diferentes tipos de AINEs, são utilizados no tratamento da dor aguda, moderada e crônica decorrente de processo inflamatório (2). Os fármacos antiinflamatórios são classificados em duas classes totalmente diferentes uma das outras: os anti-inflamatórios esteroidais que são denominados de corticosteroides (AIEs) são fármacos a base estrutural de hormônios (colesterol), o que não acontece com as estruturas dos AINEs (3).

O mecanismo de ação dos AINEs é pelo bloqueio das enzimas COXs, evitando que haja a produção das $P G s$ e dos $\mathrm{TX}_{2}$, que são os principais mediadores do processo inflamatório impedindo que ocorra o processo inflamatório inibindo (4).

Atualmente são conhecidas três isoformas das enzimas COXs: COX-1, COX-2 e COX-3 ${ }^{(5)}$. A isoforma COX-1 é demonstrada constitutivamente em vários tecidos, como, por exemplo: rins, coração, plaquetas e estômago e, está ligada juntamente a sinalização que ocorre entre as células e no equilíbrio tecidual (6). A isoforma COX-2 ocorre por um mecanismo de indução atuando principalmente nas células do processo inflamatório, pois quando estas células são ativadas durante processo da inflamação, elas fazem com que facilita a resposta inflamatória (6).

Já a isoforma COX-3, no entanto, é uma variante da isoforma COX-1 ocorrido através de um splicing alternativo, essa isoforma é encontrada em abundância no coração e no córtex cerebral, há pesquisas 
afim de obter mais informações a respeito das suas funções e modulações ${ }^{(7)}$.

\section{MATERIAIS E MÉTODOS}

O presente estudo trata-se de uma revisão bibliográfica, realizado no período de jan. de 2018 a mar. 2018, por meio de levantamento em livros, monografia, tese, dissertações e artigos científicos, disponíveis nas bases de dados, como: Biblioteca Virtual em Saúde (BVS), Scientific Eletronic Library (SCIELO). Os critérios de inclusão foram periódicos publicados entre os anos de 2000-2017, coerentes com os temas pesquisados disponíveis nas plataformas científicas. Os critérios de exclusão foram periódicos sem fundamentação cientifica, blogs, páginas da internet e periódicos inferiores ao ano de 2000.

\section{REVISÃO DE LITERATURA}

\subsection{História dos AINES}

A história dos AINEs já é conhecida há muito tempo. Os povos antigos do Egito já faziam o uso do mirtilo e a casca de salgueiro para 0 tratamento da dor reumática, assim como estava descrito no papiro de Ebers, sendo considerado um dos mais antigos texto médico descrito sobre o uso de soluções de plantas no tratamento da dor e inflamação ${ }^{(8)}$.

Com o passar do tempo, Hipocrates (460-377 a. C.) instruía o uso da casca do salgueiro para o alívio da dor e da febre. No ano de 1828 , a salicina foi isolada da casca de salgueiro (Salix Alba) por Johann Andreas Buchner, após 10 anos, Rafaelle Piria fez com que deste composto produzirse o ácido salicílico, que também podia ser encontrado em várias espécies de Spiraea, nomeadamente Spiraea ulmaria. Posteriormente, Hammond Kolbe sintetizou o ácido salicílico, que começou a ser produzido industrialmente em $1874{ }^{(8)}$.

No ano de 1897, Felix Hoffman, funcionário da Bayer ${ }^{\circledR}$, decidiu acetilar 0 ácido salicílico após seu pai ter feito o uso ácido salicílico para o tratamento da artrite e logo após ter reclamado do gosto amargo. Surge assim o ácido acetilsalicílico (AAS), o primeiro fármaco anti-inflamatório não esteróide, que começou a ser comercializado como Aspirina ${ }^{\circledR}$, pela Bayer®, em 1899 (8)

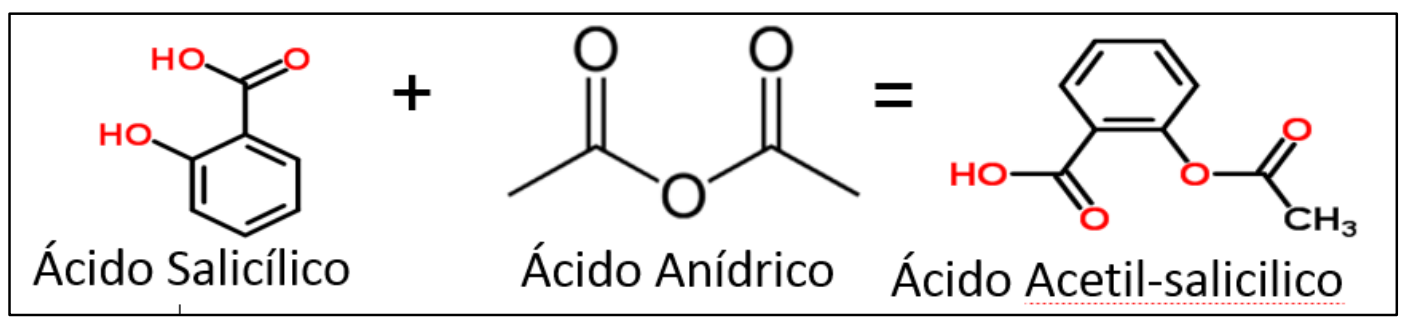


Porém devido esses fármacos serem acompanhados de vários efeitos colaterais ocorridos principalmente sobre o Trato Gastrointestinal (TGI), o que desencadeou novas pesquisas para sintetizar substâncias com menores efeitos colaterais e, no ano de 1950 foi produzido o primeiro fármaco da classe de anti-inflamatório não salicilato a Fenilbutazona ${ }^{(9)}$.

Mas, observou-se que este fármaco levou o aparecimento de casos de agranulocitose, o que levou rapidamente ao seu abandono, sendo assim pouco utilizado ${ }^{9}$. No ano de 1963 surge mais um anti-inflamatório a Indometacina, outro composto não salicilado, com grandes ações analgésicas e anti-inflamatória, desenvolvida com o intuito de substituir a Fenilbutazona. E, a partir daí novos fármacos acídicos ou não, passaram a serem produzidos, procurando-se encontrar cada vez mais fármacos com eficácia e menos efeitos colaterais, principalmente sobre o TGI e, dentre eles estão: aceclofenaco, nimesulida, meloxicam, sulindaco naproxeno ibuprofeno, fentiazaco, tenoxicam, diclofenaco, cetoprofeno, piroxicam, dentre outros. Porem estes nos dias atuais é denominado de AINEs tradicionais ou convencionais ${ }^{(9)}$.

\subsection{Fisiologia da Inflamação}

A inflamação ocorre através de um mecanismo natural de defesa desencadeado pelo nosso organismo quando Ihe são infligidas agressões/danos de natureza física, química ou infecciosa. $O$ processo inflamatório tem como principal função delimitar a área afetada permitindo que esta possa ser convenientemente reparada e regenerada (10-11).

A inflamação faz com que aumenta a liberação de PGs que agem sensibilizam os terminais periféricos dos nociceptores e aumenta a produção da hipersensibilidade à dor localizada. Descobriram que as PGs alem de sensibiizar os nocepptores periféricos, elas podem agir no SNC produzindo hiperalgesia ${ }^{(12)}$.

O sistema fisiológico envolve uma ação em conjunto com o sistema imunológico e o tecido no qual tenha ocorrido uma lesão. A resposta inflamatória é importantíssima para a sobrevivência, pois atua protegendo o organismo de estímulos nocivos. Em determinadas situações e doenças, essa resposta pode se complicar tornando-a excessiva, sem qualquer benefício ao organismo e com sérios tipos de efeitos colaterais ${ }^{(13)}$. A inflamação é um termo geral usado para as mudanças que pode haver ocorrido nos tecidos vascularizados como resposta a dano tecidual causado por bactérias, substâncias químicas, reações 
imunológicas, trauma ou qualquer outro fator. São vários tipos de mediadores químicos envolvidos no desenvolvimento do processo inflamatório, podendo ser de origem tissular, como as aminas vasoativas (13-14).

Os fenômenos irritativos são constitutivos de um conjunto de modificações provocadas pelo agente causador da inflamação que resulta na liberação de mediadores químicos responsável pelo processo inflamatório (15).

As prostaglandinas estão envolvidas em diferentes processos fisiológicos e patológicos, incluindo vasodilatação ou vasoconstrição, contração ou relaxamento da musculatura brônquica ou uterina, hipotensão, ovulação, metabolismo ósseo, aumento do fluxo sanguíneo renal resultando em diurese, natriurese, caliurese e estímulo de secreção de renina, inibição da secreção gástrica de ácido, resposta imunológica, hiperalgesia, regulação da atividade quimiotática celular, resposta endócrina e angiogênese, entre outros ${ }^{(16)}$.

\subsection{Classificações das Cicloxigenases} (COXs)

As isoformas da COXs são sintetizadas a partir da degradação do Ácido Araquidônico (AA), assim essas enzimas possuem um papel importantíssimo na síntese de prostanóides
$\left(\mathrm{PGl}_{2}, \mathrm{PGD}_{2}, \mathrm{PGE}_{2}, \mathrm{PGF}_{2}\right)$ e $\mathrm{TXA}_{2}$, que exercem ação de vasoconstritor. (17)

AA é liberado a partir da degradação dos fosfolipídios de membrana, na ativação celular e sob ação da enzima fosfolipase A2 a sua metabolização ocorre por diferentes vias, via cicloxigenase COX-1, COX-2 e COX-3 e a via lipoxigenase (LO) ${ }^{17-18}$. As COXs são codificadas por dois tipos de genes, sendo que a COX-1 e COX-3 codificadas pelo gene 1 e a COX-2 convertida pelo gene 2 da COX.

A primeira isoforma da COX, a COX1, é denominada constitutiva ou fisiológica, a qual tem papel fundamental nos processos fisiológicos do organismo, sendo encontrada praticamente em todos tecidos, exercendo várias funções, como: atividade plaquetária, reprodutiva, pulmonares, renais, no sistema nervoso central, na produção de muco e mucosa gastrointestinal, resposta autoimunes e reduzindo os riscos cardiovasculares. São encarregadas da síntese de PGs (2).

A segunda isoforma da COX, a COX 2, é considerada induzível a Cox 2 está presente nos locais de inflamação, sendo, por isso, denominada de enzima indutiva. Ela é expressa primeiramente por células envolvidas no processo inflamatório, como macrófagos, monócitos e sinoviócitos, e as citosinas e endotoxinas, sendo os mediadores de edemas, também 
encontrada em células cardiovasculares natural, útero, cérebro, ovários, rins e medula espinhal, entre outros ${ }^{(10)}$.

A COX-3 é uma variação ou análogo da COX 1, podendo ser localizada no SNC. Podendo ser encontrada em diferentes tecidos, como, na aorta e no coração, pois é uma enzima que tem ação inibitória de drogas antipiréticas analgésicas e potencialmente inibidas pelos AINEs, essa inibição pode ocorre um processo primário onde essas drogas podem diminuir a dor $\mathrm{e}$ possivelmente a febre ${ }^{(18-19)}$.

Figura 1 -Mediadores químicos gerados pelo ácido araquidônico. Adaptado. (Katzung,2005).COX (Cicloxigenase), AA (Ácido Araquidônico).

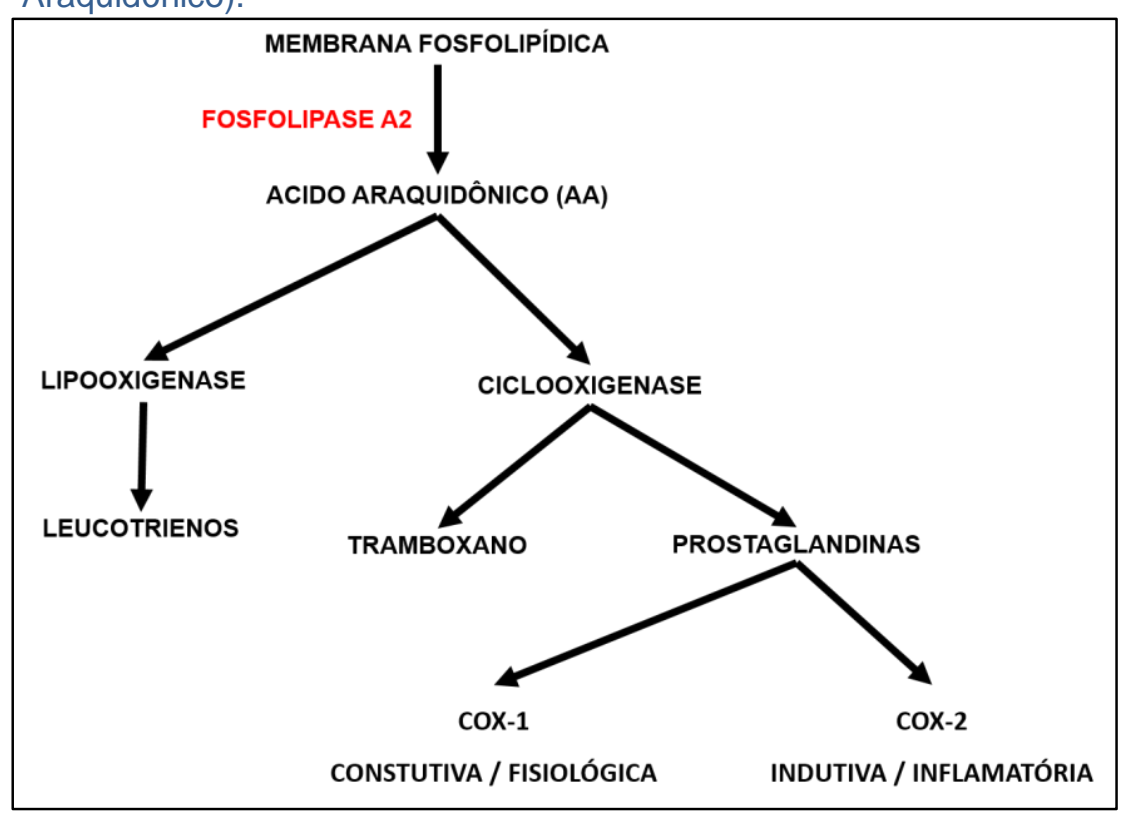

\subsection{Classificações dos fármacos}

Existem duas classes de fármacos inibidores que são seletivos e os fármacos não seletivos das COXs $1-2$. Os inibidores da COX-2 é uma classe de fármacos potentes e, habitualmente, possuem menos efeitos adversos, principalmente em relação ao TGI ${ }^{(20)}$. Derivado ácido salicílico é um inibidor irreversível da COX-1 e a COX-2, mas apresentam seletividade para a COX1. Já os fármacos sintetizados do ácido acético inibem a produção de $\mathrm{PGs}$ de modo reversível (21-22). Conforme a ação de todos os fármacos sobre a COX-1 e COX2, os AINEs podem ser classificados em três grupos: o primeiro é conhecido como inibidores potentes da COX-1 tais como o ácido acetilsalicílico, ibuprofeno e indometacina; o segundo são os inibidores seletivos da COX-2, porem possuem atividade de inibição sobre a COX-1 tais como piroxicam e diclofenaco; e o ultimo grupo são os inibidores específicos da COX-2, não agem sobre a COX-1 como, por exemplo: celecoxibe, rofecoxibe, etoricoxibe, dentre outros ${ }^{(20)}$. 
Quadro 1 - Classificação dos AINEs.

\section{CLASSIFICÇÃO DOS AINES}

\begin{tabular}{|c|c|c|c|}
\hline $\begin{array}{c}\text { Inibidores Seletivos } \\
\text { COX-1 }\end{array}$ & $\begin{array}{c}\text { Inibidores Não } \\
\text { Seletivos COX-1 }\end{array}$ & $\begin{array}{c}\text { Inibidores Não } \\
\text { Seletivos COX-1 } \\
\text { COX-2 }\end{array}$ & $\begin{array}{c}\text { Inibidores Altamente } \\
\text { Seletivos COX-2 }\end{array}$ \\
\hline AAS $(<100 \mathrm{mg})$ & Indometacina & Nimesulida & Valdecoxibe (Bextra) \\
\hline & Piroxicam & Etodolaco & Celecoxibe (Celebra) \\
\hline & Diclofenaco & & Lumiracoxibe (Prexige) \\
\hline & Ibuprofeno & & Etoricoxibe (Arcoxia) \\
\hline & & & Parecoxibe \\
\hline
\end{tabular}

Fonte: Adaptado BATLOUNI, M.,2010.

\subsection{Mecanismos de ação dos AINEs}

A ação primária do AAS é a inativação da COX por acetilação irreversível da prostaglandina sintase. Sendo que a prostaglandina sintase é a enzima catalizadora da primeira fase $\mathrm{da}$ biossíntese da prostaglandina, a partir do AA (23).

Quando ocorre uma determinada agressão que atinge as membranas celulares, desencadeia-se uma cascata de reações que culminam com a formação de tromboxanos e prostaglandinas, designados globalmente por prostanóides. Após esta lesão, algumas citocinas inflamatórias, como a interleucina-1, ativam a fosfolipase A2, uma enzima presente nos leucócitos e nas plaquetas, que irá degradar os fosfolipídios presentes nas membranas, o que dará origem a um ácido graxo, o ácido araquidônico (10).

As principais ações dos AINEs estão relacionadas à capacidade de impedir que a enzima COXs realize a hidrolise do AA em PGs e Prostaciclinas, composto que fazem parte do processo inflamatório e ligada a sensibilização das unidades dolorosas centrais e periféricas (24-25). Quando os AINEs bloqueiam as enzimas COXs, esse efeito contribui para vários efeitos colaterais, como por exemplo: impedem que ocorra a ação das PGs no efeito de vasodilatador, estimulando o efeito de vasoconstricção renal e diminuindo a taxa de filtração glomerular, levando a causar necrose tubular aguda, inibem a ação das 
PGs sobre os linfócitos $T$, fazendo com que ocorra a ativação dessas células, consequentemente aumentando a liberação de citocinas pró- inflamatória, movimenta o AA para a via das lipoxigenases, expandindo a síntese de leucotrienos pró- inflamatórios e faz com que a enzima lipoxigenase induz a permeabilidade capilar, causando assim proteinúria por alterar a barreira de filtração glomerular (24).

\subsection{Farmacocinética}

Esses fármacos são administrados tanto por via oral, ou endovenosa, retal e transdermica (26). Todos os AINEs têm apresentado uma ótima absorção TGl e retal, chegando à meia vida plasmática de duas a três horas após ser administrado. A absorção desses medicamentos pelo TGl superior pode ser comprometida com uso concomitante com outros fármacos, alimentos (26). Logo após a absorção desses fármacos eles são ligados a proteínas plasmáticas na corrente sanguínea, a fração livre desses fármacos metabolicamente ativa representa a menos de $1 \%$ da fração sérica e se movimenta na forma de ácidos. Uma grande concentração pode ser encontrada em áreas acidas como, por exemplo; estômago medula óssea e em locais com processos inflamatórios. Os AINEs conseguem atravessar a barreira hematoencefalica, agindo diretamente SNC e na placenta, causando efeitos adversos durante a gravidez (26).

Os AINEs na maioria das vezes são ácidos fracos bem absorvidos no TGl, com a concentração aproximadamente de 1 - 4 horas. Com ligação as proteínas plasmáticas de 95-99\% e ocorre a biotransformação hepática e a sua excreção pela via renal (27).

\subsection{Efeitos farmacológicos}

Todos os AINEs têm a sua ação antipirética, analgésica e anti-inflamatória, exceto 0 paracetamol que tem ação antipirética e analgésica, mas praticamente não possui atividade anti-inflamatória (27).

O uso do AAS faz com que ocorra a inibição da síntese PGs que estão especialmente associadas com 0 desenvolvimento da dor que acompanha a lesão e inflamação, abaixando também, a febre por dilatação dos vasos sanguíneos periféricos e aumentando a dissipação do calor por transpiração (23).

Os AINEs possuem três ações principais: ação anti-inflamatória, ação analgésica e ação antipirética. Sua ação anti-inflamatória está claramente vinculada à inibição da $\operatorname{COX} 2$, resultando normalmente em vasodilatação, edema de modo indireto, e dor. É provável que quando utilizados para casos inflamatórios, 
seus efeitos indesejáveis decorram em grande parte da inibição da $\operatorname{COX} 1{ }^{(28)}$.

Inibindo as COXs os AINEs podem provocar uma série de efeitos colaterais como: diarreia, hemorragia gastrointestinal, dispepsia, úlcera péptica, disfunção e falência renal, inibição da agregação plaquetária e aumento do tempo de sangramento, alterações dos testes de função renal, icterícia e interações com outras drogas (28).

\section{CONSIDERAÇÕES FINAIS}

AINEs são uma excelente classe de fármacos para tratar os efeitos indesejáveis causados pela resposta inflamatória. Eles agem diminuindo o edema, a hiperemia, a febre, e a dor, melhorando a condição de qualidade de vida do paciente. Os AINEs apresentam uma segurança significativa, porem eles podem apresentar vários efeitos adversos, que podem variar desde uma dispepsia até a morte causada por uma ulcera perfurada ou hemorragia. Seu uso, portanto, deve ser seguro, para que possa oferecer mais benefícios do que risco ao paciente. Sua administração sempre deve ter um acompanhamento de um profissional com exames laboratoriais.

\section{REFERÊNCIAS}

1. Santon KLM. Anti-inflamatórios não esteroides: agentes terapêuticos no processo inflamatório e principais consequências do uso indiscriminado. [Acesso em: 13 de out. de 2017]. Disponível em:<https://editorarealize.com.br/revistas/c onbracis/trabalhos/TRABALHO_EV055_M D4_SA11_ID968_14052016125359.pdf>

2. Batlouni M. Anti-Inflamatórios Não Esteroides: Efeitos Cardiovasculares, CérebroVasculares e Renais. Instituto Dante Pazzanese de Cardiologia, São Paulo, SP - Brasil Arq Bras Cardiol 2010;94(4): 556-563. [Acesso em: 10 de out. de 2017]. Disponíveis em:< http://www.scielo.br/pdf/abc/v94n4/v94n4a 19>

3. Filho CCV, Silva DA. Avaliação da dispensação de fármacos parcialmente seletivos para COX-2. Acta Biomédica Brasiliensia / Volume 4/ ํㅡ 2/ Julho de 2013. [Acesso em: 15 de set. 2017]. Disponível em:<file:///D:/Windows\%207/Downloads/Di
alnetAvaliacaoDaDispensacaoDeFarmaco sParcialmenteSeleti-4713406.pdf>

4. Pancote CG. Planejamento, síntese e avaliação biológica de derivados pirrólicos com potencial atividade anti-inflamatória. Universidade de São Paulo Faculdade de Ciências Farmacêuticas. São Paulo 2009. [Acesso em: 01 de out. de 2017]. Disponível em:<file:///D:/Windows\%207/Downloads/te se_pancote.pdf>

5. COSTA DLS. Papel da inflamação no desenvolvimento tumoral - potencial terapêutico dos inibidores da cox-2. Faculdade de Medicina da Universidade de Coimbra. março - 2010. [Acesso em: 20 de out. de 2017]. Disponível em:< https://estudogeral.sib.uc.pt/bitstream/1031 6/26060/3/Tese\%20de\%20Mestrado\%20\%20final.pdf

6. Ely LS. Estudo dos anti-inflamatórios e analgésicos utilizados pelos idosos da Estratégia Saúde da Família de Porto Alegre. Universidade Católica do Rio Grande do Sul. Porto Alegre - RS 2014. [Acesso em: 01 de set de 2017]. Disponível 
em:

http://repositorio.pucrs.br/dspace/bitstream /10923/6863/1/000462079-

Texto\%2BCompleto-0.pdf

7. Coutinho MAS, Muzitano MF, Costa SS. Flavonoides: Potenciais agentes terapêuticos para o processo inflamatório. Rev Virtual Quim. 2009;1(3):241-56. [Acesso em: 01 de set. de 2017]. Disponível em:< http://rvqsub.sbq.org.br/index.php/rvq/article/view/5 1>

8. Tavares TIA. Riscos e benefícios dos anti-inflamatórios não esteróides inibidores seletivos da ciclo-oxigenase 2. Universidade Fernando Pessoa Faculdade de Ciências da Saúde Ciências Farmacêuticas Porto, 2012. [Acesso em: 02denov.2017]. Disponívelem:< https://bdigital.ufp.pt/bitstream/10284/3749/ 3/PPG_TaniaTavares.pdf>

9. Monteiro ECA et al. Os Anti-inflamatórios não esteroidais (AINEs). Revista de Reumatologia clínica Maio 08 v9 n2. [Acesso em: 12 de out. de 2017]. Disponível em:

http://www.moreirajr.com.br/revistas.asp?f ase $=r 003 \& i d$ materia $=3744$

10. Verdasca ACRS. "Utilização dos AntiInflamatórios Não Esteróides (AINES) em Medicina Dentária: Indicações, ContraIndicações e Efeitos Adversos". Faculdade de Medicina Dentaria. UNI. Porto-Julh2015. [Acesso em 03 de nov. de 2017]. Disponível em:

file://C:/Users/Gleicielle/Downloads/Ana_C lara_Verdasca\%20(1).pdf

11. Silva SAM, Gonçalves FF, Macedo CC. Síntese e Caracterização fisíca de hidrogéis de poli (2 -Hidroxietilmetacrilato) Contendo Diclofenaco de Sódio. [Acesso em: 01 de set. 2017]. Disponível em:http://www.fepi.br/revista/index.php/revi sta/article/view/222>

12. Hinz B, Brune K. Cyclooxygenase-210 years later. Journal of Pharmacology and Experimental Therapeutics, v. 300, n. 2, p. 367-375, 2002.

13. Pires PA. Potencial Analgésico, Antiedematogênico,Antipirético e Atividade Ulcerogênica de FármacosAntiinflamatórios em Roedores. Universidade Federal Rural do Rio de Janeiro. Instituto de Veterinária. Seropédica, RJ. Agosto de 2009. [Acesso em: 05 de out. 2017]. Disponível em: http://r1.ufrrj.br/wp/ppgmv/files/2011/01/pir es-2.pdf

14. Silva AA. Avaliação Clínica de Rattus norvegicus Após Terapia Anti-inflatória com Inibidor Seletivo ou não para COX-2 por Extrapolação Alométrica. Universidade de Santa Maria - SC. 02 de Agosto de 2004. [Acesso em: 05 de nov. 2017]. Disponível em:<

http://repositorio.ufsm.br/bitstream/handle/ 1/4112/tesefinal.pdf>

15. Martins FI. Planejamento, síntese e avaliação farmacológica de novos candidatos a protótipos de fármacos antiinflamatórios desenhados a partir do nerolidilcatecol. Universidade Federal de Goiás - GO. 2009. [Acesso em: 05 de nov. 2017]. Disponível em: https://repositorio.bc.ufg.br/tede/bitstream/t de/2131/1/Dissertacao\%20Fabiula.pdf

16. Kummer CL. Antiinflamatórios não esteróides inibidores da ciclooxigenase2(COX-2): aspectos atuais. Revista Brasileira De Anestesiologia. Volume 52. Campinas 2002. [Acesso em: 06 de nov 2017]. Disponível em: http://www.scielo.br/pdf/rba/v52n4/v52n4a1 4.pdf

17. Varalda DB, Motta AA. Artigo de revisão Reações adversas aos antiinflamatórios não esteroidais. Rev. bras. alerg. imunopatol. 2009; 32(1):27-34. [Acesso em: 06 de nov de 2017]. Disponível em:< http://www.asbai.org.br/revistas/Vol321/AR T\%20109\%20\%20Rea\%C3\%A7\%C3\%B5 es\%20adversas\%20aos\%20antiinflamat\%C3\%B3rios.pdf> 
18. Mosquini AF et al. características farmacológicas dos antiinflamatórios não esteroidais - revisão de literatura. REVISÃO CIENTIFICA ELETRÔNICA DE MEDICINA VETERINÁRIA. Julho de 2011. [Acesso em: 06 de nov 2017]. Disponível em:

https://mail.google.com/mail/u/0/\#inbox/16 20 cadcce $460 \mathrm{~b} 81$ ? projector $=1$ \& message $\mathrm{Pa}$ rtld $=0.6$

19. Luiz JJ et al. Uso de anti-inflamatórios não-hormonais durante a amamentação: quais podem ser utilizados? Rev Paul Pediatria 2006;24(2):171-9. [Acesso em: 06 de nov 2017]. Disponível em:< http://www.redalyc.org/pdf/4060/40603891 6013.pdf>

20. Beirão ACRV. Interações medicamentosas dos Anti Inflamatórios Não Esteróides (AINEs). Faculdade de Medicina Dentária Universidade do Porto. 2016. [Acesso em: 06 de nov de 2017]. Disponível em:< https://sigarra.up.pt/fmdup/pt/pub_geral.sh ow_file?pi_gdoc_id=934671>

21. Catella-Lawson F et al. Cyclooxygenase inhibitors and the antiplatelet effects of aspirin. New England Journal of Medicine, v. 345, n. 25, p. 1809-1817, 2001.

22. Antunes AL, Albino AJS, Guedes NEF, Castro GFP. Efeito antiplaquetário do ácido acetilsalicílico em prevenção secundária do infarto agudo do miocárdio. Revista transformar, 8(8), 179-192. [Acesso em: 15 de nov. 2017]. Disponível em: file://C:/Users/Gleicielle/Desktop/AINES_R EVISAO_01.pdf

23. Melgaço SSC et al. Nefrotoxicidade dos anti-inflamatórios não esteroidais. Medicina (Ribeirão Preto) 2010; 43(4): 382-90. [Acesso em: 01 de out. 2017]. Disponível em: $<$

https://www.revistas.usp.br/rmrp/article/vie wFile/188/189>

24. Castel BMM et al. As bases farmacológicas dos cuidados farmacêuticos: o caso dos AINEs. Acta
Farmacêutica Portuguesa 2013, vol. 2, n. 2, pp. 79-87. [Acesso em: 02denov.2017]. Disponível em:< http://www.actafarmaceuticaportuguesa.co m/index.php/afp/article/view/3/10>

25. Trevisan, VFM, Fidelix TSA, Appenzeller S. Uso dos anti-inflamatórios não hormonais na artrite reumatoide, osteoartrite e na lombalgia. RBM Jan/Fev 12 v 69 n1/2. [Acesso em: 05 de nov. 2017]. Disponível em:< http://www.moreirajr.com.br/revistas.asp?id _materia $=4960 \& \mathrm{fase}=$ imprime

26. Muri EMF, Sposito MMM, Metsavaht L. Anti-inflamatórios não-esteroidais e sua farmacologia local. Revista Acta Fisíatrica Dezembro de 2009, v16. N4. [Acesso em: 12 out. de 2017]. Disponível em:< http://www.actafisiatrica.org.br/detalhe_arti go.asp?id=92>

27. Silva JM, Mendonça PP, Partata AK. Anti-inflamatórios não esteroidais e suas propriedades gerais. Revista Científica do ITPAC, Araguaína, v.7, n.4, Pub.5, Outubro2014. [Acesso em: 15 de nov. 2017].

Disponívelem:<https://assets.itpac.br/arqui vos/Revista/74/artigo5.pdf>http://www.site. ajes.edu.br/mostra/arquivos/20170404164 250.pdf

28. Schallemberger JB, Pletsch MU. Riscos do uso indiscriminado de anti-inflamatórios não esteroidais (AINES). Salão do Conhecimento, v. 2, n. 01, 2014. [Acesso em: 15 de nov. 2017]. Disponível em:< https://www.publicacoeseventos.unijui.edu. br/index.php/salaoconhecimento/article/vie w/3490> 


\section{Como citar (Vancouver)}

Sandoval AC, Fernandes DR, Silva EA, Terra Júnior AT. O uso indiscriminado dos anti-inflamatórios não esteroidais (AINES). Rev Cient Fac Educ e Meio Ambiente [Internet]. 2017;8(2):165-176. DOI: http://dx.doi.org/0.31072/rcf.v8i2.589 